\title{
Ultimate Resolution Limit for Z-contrast STEM: Atoms are Smaller in ADF
}

\author{
Y. Peng, M. P. Oxley, A. R. Lupini, M. F. Chisholm and S. J. Pennycook
}

Condensed Matter Sciences Division, Oak Ridge National Laboratory, Oak Ridge, TN 37831-6031

Achieving higher resolution has been a longstanding goal for electron microscopy. Steady instrumental improvements have enabled experimental observations down to the atomic scale and even to the subÅngstrom level. For example, with an aberration-corrected VG Microscope's 300 kV HB603U STEM, pairs of Si columns $0.78 \AA$ apart in Si [112] (Fig. 1a) have been directly resolved at ORNL [1]. Comparison to image simulations allows one to extract the sample thickness, probe defocus and source size using a linear regression technique [2] (Fig. 1d). There is excellent agreement between the simulated and experimental image profile (Fig. 1c), which has a dip contrast, $\left(I_{\text {column }}-I_{\text {dip }}\right) / I_{\text {column }}$, of about $20 \%$ for the Si dumbbell.

In spite of being an important issue, the criterion for the two-point resolution has not been unambiguously defined [3]. For this reason a concept of "resolution quality" was introduced and described by a Rayleigh-Sparrow parameter [4]. The point-spread function of a microscope, or the probe size in a STEM, can be reduced by instrumental improvements such as aberration correction. Thus, it is the width of the atom potential that forms the ultimate limit on the resolution. In ADF STEM imaging, however, the effective potential of the atom can be adjusted by the detector. For a weakly scattering object, it has been shown [5] that the ADF detector selects only the high order Fourier components of the potential, and the incoherent image is given by a convolution between the high pass filtered potential and the point-spread function. In bright field STEM or TEM imaging, the effective potential is the elastic potential (Fig. 2a), while for Z-contrast imaging the effective potential is the inelastic potential filtered by the ADF detector. The ADF potential has a much reduced background and sharper peaks due to the high pass filtering (Fig. 2b). Increasing the ADF detector inner angle gives rise to even narrower potential peaks (Fig. 2c), effectively making the atoms smaller. With adjustable object size, we immediately see the resolution advantage of ADF STEM over TEM. Thermal vibration has a broadening effect on the size of the atoms (Fig. 2), and this blurring of the atom potential cannot be reduced by any filtering, only by reducing the specimen temperature.

As can be analytically shown [2], the reflection distribution in a STEM diffractogram depends on the lens aberration function and specimen, whereas the highest transferred spatial frequency is decided by the probe-forming aperture diameter only (Fig. 3a). The cutoff frequency is often called the diffraction limit to resolution, but one should bear in mind that, due to probe aberrations, for example, higher frequency transfer in the image is just a necessary but not sufficient condition for the achievement of higher resolution. Also noise will impose a limit on the resolution. Fig. $3 b$ shows the normalized noise histogram derived from the experimental $\mathrm{Si}[112]$ diffractogram. The probability of a reflection to be a real signal can be evaluated in terms of a confidence level by the cumulative probability corresponding to its intensity (Fig. 3c). The 173 reflection at $0.71 \AA$ has a $99 \%$ confidence level, the 555 reflection at $0.63 \AA 91 \%$, while the 804 reflection at $0.61 \AA$ has a confidence level of $76 \%$ [6].

\section{References:}

[1] P. D. Nellist et al., Science 305 (2004) 1741. [2] Y. Peng et al., in preparation.

[3] A. J. den Dekker and A. van den Bos, J. Opt. Soc. Am. A 14 (1997) 547.

[4] M. A. O’Keefe, L. F. Allard and D. A. Blom, J. Electron Microsc. 54 (2005) 169. 
[5] S. J. Pennycook and P. D. Nellist (1999). In "Impact of Electron and Scanning Probe Microscopy on Materials Research", pp. 161-207. Kluwer Academic Publishers.

[6] This research was sponsored by DOE BES Division of Materials Sciences under contract DEAC05-00OR22725 with ORNL managed and operated by UT-Battelle, LLC.
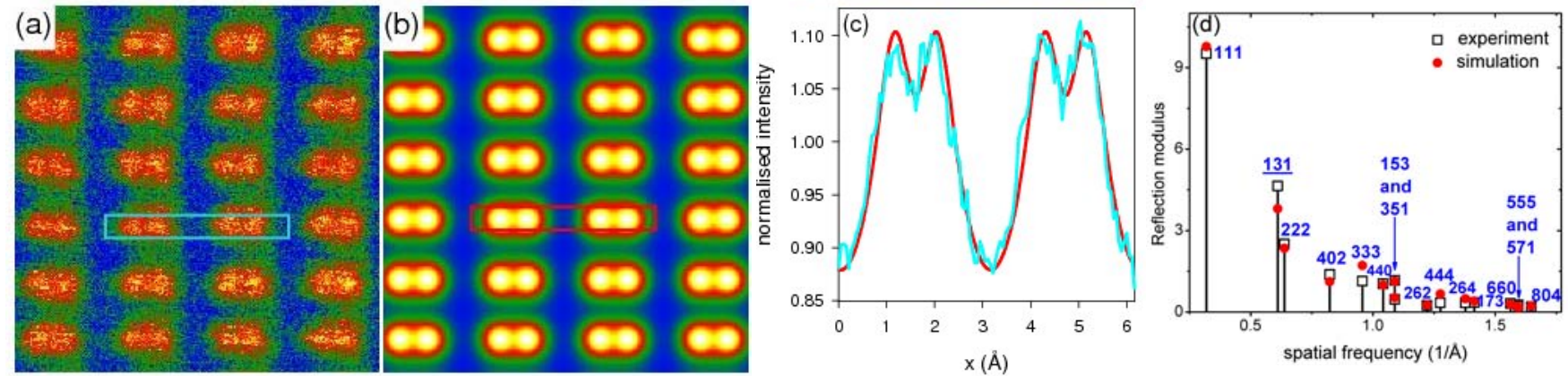

FIG. 1: (a) Experimental Z-contrast image of Si[112] (has been unwarped to remove image drift). (b) Simulated image with parameters: probe semiangle $=22 \mathrm{mrad}, \mathrm{Cs}=-0.037 \mathrm{~mm}, \mathrm{C} 5=100 \mathrm{~mm}$, ADF detector $=90-200 \mathrm{mrad}$, defocus $=-35 \AA$, sample thickness $=250 \AA$. The source size is included by a convolution with a Gaussian of $0.45 \AA$ FWHM. (c) Experimental and simulated profiles resolving the $0.78 \AA$ dumbbell. (d) Best fit between simulated and experimental reflections in the diffractogram.
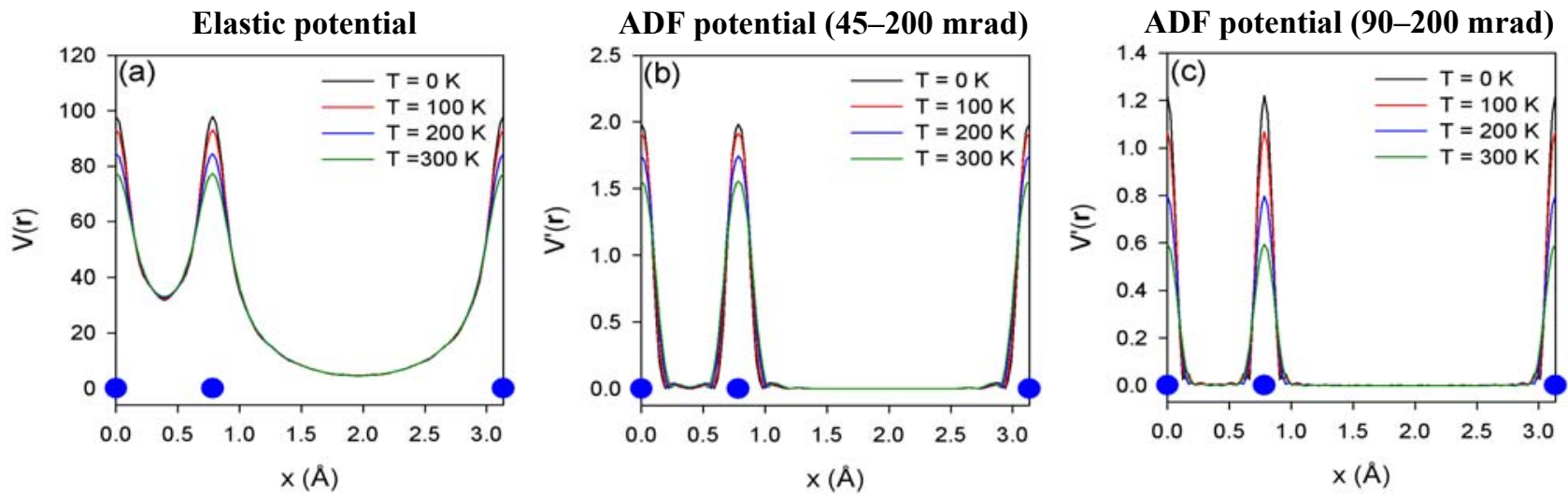

FIG. 2: Profiles of projected Si[112] crystal potentials at different temperatures. (a) Elastic potential, and inelastic potential filtered by an ADF detector of (b) 45-200 mrad and (c) 90-200 mrad.
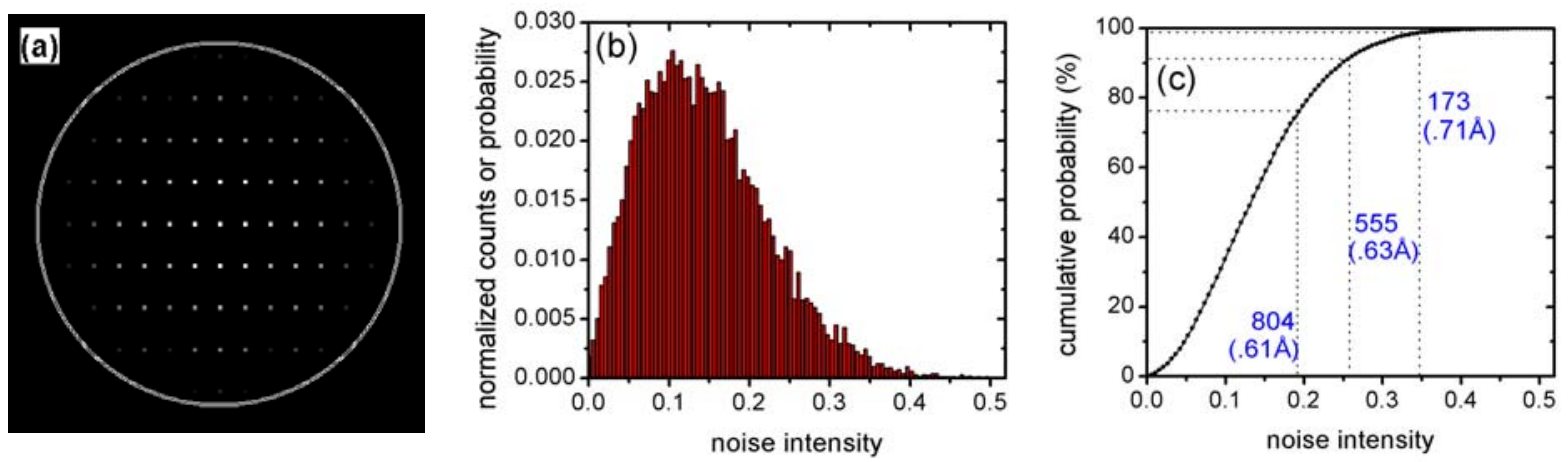

FIG. 3: (a) Denoted by a circle, the objective aperture defines the limit to the frequency spots in a simulated diffractogram, and there is no spurious sum or difference frequencies occurring in the diffractogram. (b) Noise histogram extracted from the experimental Si[112] diffractogram. (c) Cumulative noise distribution of (b) used to estimate the confidence level of different reflections. 